\section{Cover Glass Perspectives}

Gary W. Gill, Diagnostic Cytology Laboratories, Inc.

Cover glasses, and by extension mounting media, are the de facto front lens of every microscope objective. Those who coverslip are, in effect, completing the assembly of a microscope objective with every cover glass they apply. So while coversliping is a tedious process, its visibly appreciable contribution should not be minimized.

There are optical reasons on which coverslipping do's and don'ts are based. It is optically and physically impossible for alternatives such as liquid substitutes and plastic film to duplicate the specifications and performance of glass cover glasses. Such substitutes are used, however, perhaps because there is so little related useful technical information available to discourage such use. There is more to a cover glass than meets the eye and the pocketbook, even though cover glasses cost 15 times more than slides, ounce for ounce.

According to ASTM specifications, cover glasses should have a refractive index of $1.523 \pm 0.005$, a dispersion value of 52.0 , fall within precise thickness ranges, be planoparallel, and free of optical pits and imperfections. Lens designers have long incorporated such data into their calculations, but it was not until 1953 that Setterington gathered the proprietary optical specifications used by various microscope manufacturers and proposed a uniform standard (The Specification of a Standard Microscope Cover Glass. J R. Micr. Soc. 1953, 73:69-76). Cover glasses were used first in 1789 and first made commercially in 1840 by Chance Brothers of Birmingham, England.

Engraved on each microscope objective is " 0.17 ", among other information. This number represents the thickness of cover glass for which the objective is designed to be used. Strictly speaking, this thickness is included in the thickness range of No.11/2 cover glasses. Unfortunately, however, some well intended individuals interpret this number strictly and recommend using No. $11 / 2$ cover glasses. They fail to consider the substantial and variable thickness of mounting medium and its optical consequences. In practice, use No. 1 cover ? glasses with as little mounting medium as the preparation will allow. Parenthetically, mounting media are an entire topic of their own.

A well prepared and mounted micro preparation has the potential to appear sharp and crisp at all magnifications, to be more signal than noise. Objectively speaking, the extent to which it fulfills its potential depends on a clean microscope that is adjusted to produce Koehler illumination. Less well prepared preparations can degrade image quality to degrees that range from barely perceptible to ruinous. Whether these degradations are considered problematic is a subjective matter. Some microscope users are simply insensitive to quality issues, whether by design or default.

Cover glass and mounting medium thickness should be controlled within narrow tolerances to avoid unacceptable spherical aberration, which is a geometric optics consideration. Spherical aberration means that light rays of different wavelengths arising from an object point and that pass through the same area of a lens are not brought together as a single image point.

Spherical aberration makes object images look washed out, hazy, cloudy, milky, and low in contrast. It is the same look as, and is visually indistinguishable from, that produced by glare and flare.

Glare and flare are defined as light that does not contribute to image formation, noise in other words, and produce eyestrain similar to that experienced looking through a dirty car windshield while driving into the sun. Glare arises in the object space, located before the objective, while flare arises within the image space, located inside the microscope. Glare and flare result when light falls outside the optical axis and bounces off metal parts - for example, the metal area around the objective front lens and the inside walls of the objective when the substage condenser diaphragm is opened too far or when lens surfaces are dirty.

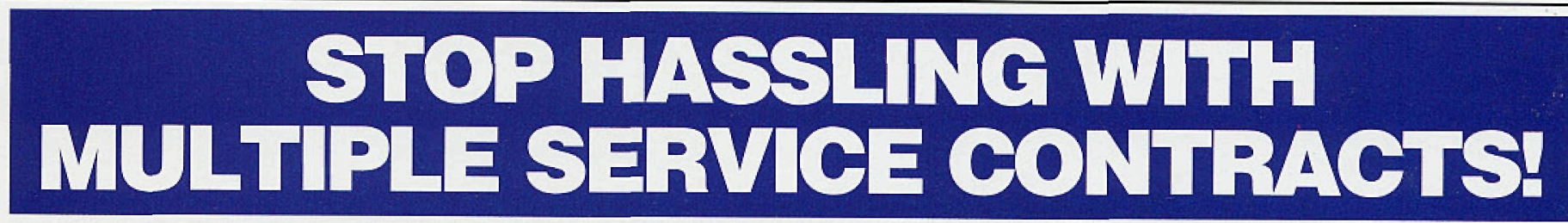

START by putting all of your instruments under one contract with MAS (regardless of make or model). Our expert EM SERVICE GROUP has the knowledge and skill to keep your instrumentation working at its best.

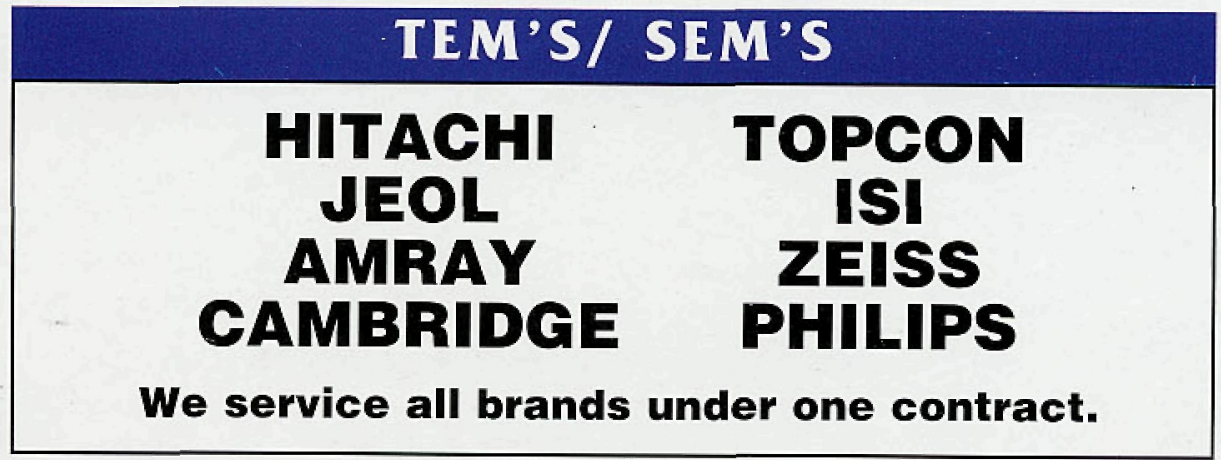

\begin{tabular}{|c|}
\hline PREP EQUIPMENT \\
\hline ULTRAMICROTOMES \\
DUPONT \& RMC \\
VACUUM COATERS \\
SPUTTER COATERS \\
MECHANICAL PUMPS \\
TURBO PUMPS \\
PLASMA ASHERS \\
\hline
\end{tabular}

WE SERVICE COMPUTER CONTROLLERS BEAM BLANKERS WATER CHILLERS
Contracts and On-Demand Emergency Service at Reasonable Rates from Factory Trained Specialists. 1-800-421-8451 616 Hutton Street • Suite $101 \cdot$ Raleigh North Carolina 27606 • 919-820-7041 FAX 770-368-8256 or ADVANCED ANALYTICAL PRODUCTS AND SERVICES 
Glare and flare look the same, and are usually referred to as simply glare, as the two are inseparable. Where glare goes, flare follows in lens systems.

Cytologic preparations are often thicker than histologic preparations and so require more stringent mounting conditions. High dry objectives with numerical apertures of 0.65 and higher are much more sensitive to cover glass and mounting medium thickness ( $\pm 15 \mu \mathrm{m}$ deviation) than $4 \mathrm{X}$ and $10 \mathrm{X}$ objectives with much lower numerical apertures. An upside down slide will be imaged satisfactorily under $10 \mathrm{X}$, but cannot be imaged at all under $40 \mathrm{X}$. Evaluating cover glass alternatives under $10 \mathrm{X}$ will result in a passing grade, but not if evaluated under $40 \mathrm{X}$ objectives.

Power for power, apochromat objectives are more sensitive to cover glass and mounting medium thickness than achromat objectives, since they have higher numerical apertures. Numerical aperture is the factor that determines thickness sensitivity. NA is a measure of the angle of the cone of light that enters an objective: the wider the cone, the greater the resolution, and the greater the optical challenge of reuniting the marginal image forming rays into sharply focused points.

Since the loss of image contrast resulting from spherical aberration and glare look identical, it is important to recognize the various contributing factors so one can systematically troubleshoot these problems.

Microscopists can recapture image quality in thick mounts by reducing the opening of the substage condenser aperture diaphragm - an optical safety net. Used with Koehler illumination, this diaphragm is imaged at the back focal plane of the objective where it controls the working numerical aperture, as opposed to the theoretical maximum NA. By thus reducing the working NA, the microscopist is increasing the objective's tolerance to thick mounts and so can image them sharply. Closing the aperture diaphragm too far, however, creates an unacceptable refractile appearance due to diffraction effects.

The use of functionally inferior cover glass alternatives is part of a larger cost-driven, ignorance-based, trend away from quality materials and methods to the benefit of the purse and at cost to the patient (in clinical settings) or quality of 0 the data. Quality outcomes are based on quality processes, not the other way around.

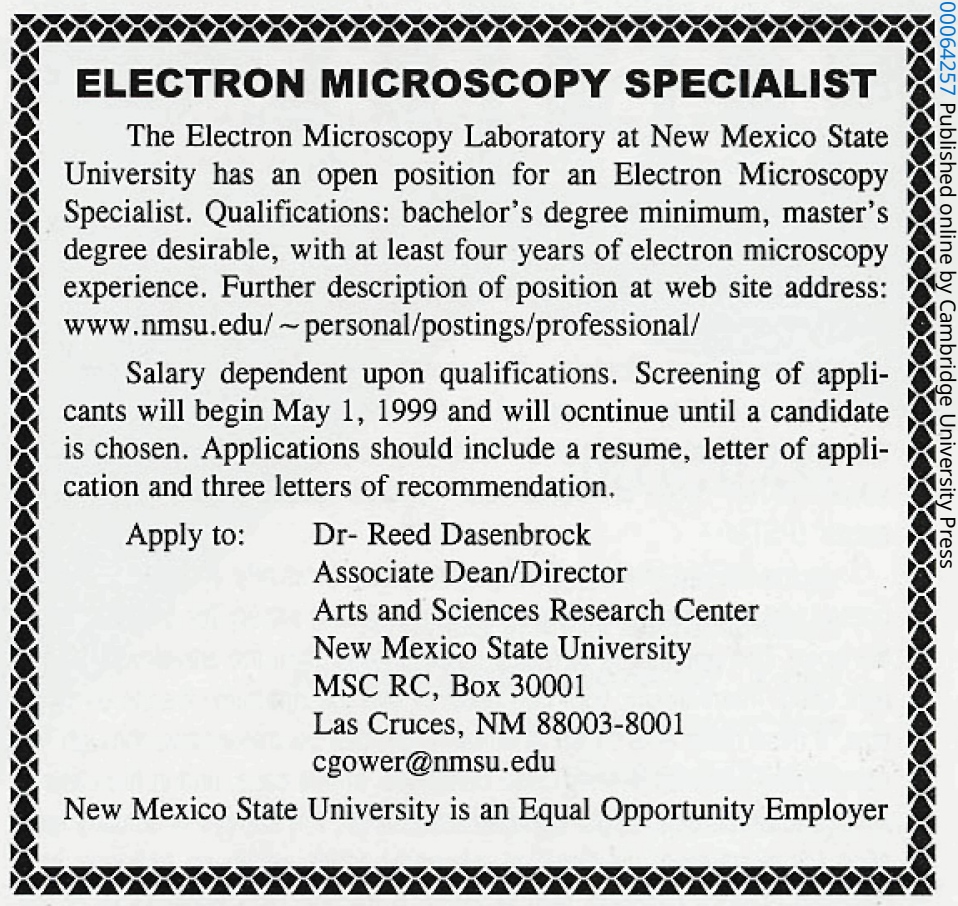

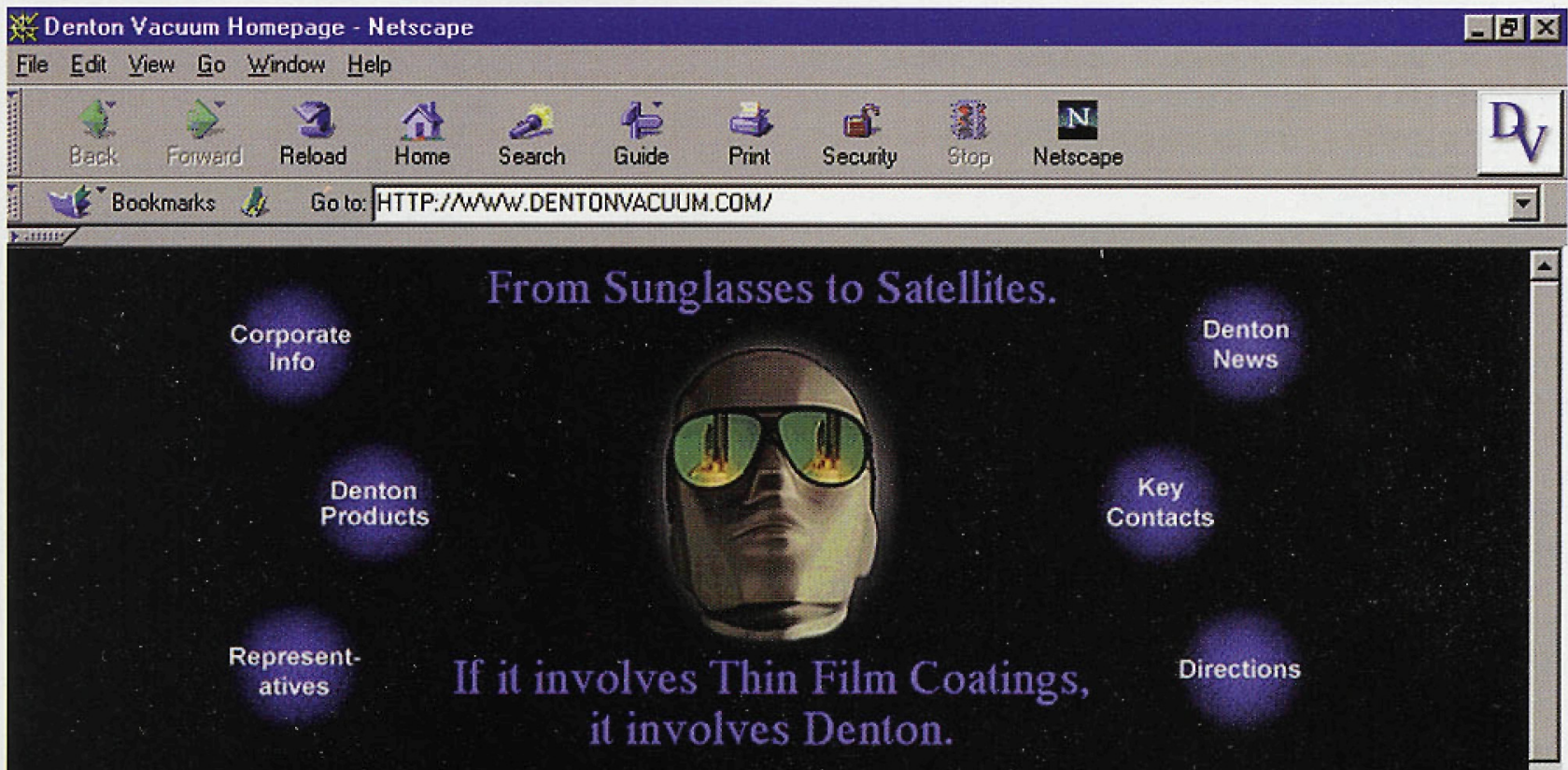

http://www.dentonvacuum.com

Desk II Cold Sputtercoater | DV 502A Carbon Coater | DV 401 Desktop Carbon Coater

HI RES 100 High Resolution Chromium Coater | DCP-1 Critical Point Dryer Desk II TSC Turbo Sputter Coater | BTT III Benchtop Carbon Coater 